\title{
BJO
}

\section{Activation of tumour-necrosis factor-related apoptosis-inducing ligand receptor enhances the severity of murine allergic conjunctivitis}

T Sumi, W Ishida, K Okumura, et al.

Br J Ophthalmol 2009 93: 110-115 originally published online September 9, 2008

doi: 10.1136/bjo.2008.144360

Updated information and services can be found at:

http://bjo.bmj.com/content/93/1/110.full.html

Topic collections Articles on similar topics can be found in the following collections

Ocular surface (379 articles)

Notes

To order reprints of this article go to:

http://bjo.bmj.com/cgi/reprintform

To subscribe to British Journal of Ophthalmology go to:

http://bjo.bmj.com/subscriptions 


\title{
Activation of tumour-necrosis factor-related apoptosis-inducing ligand receptor enhances the severity of murine allergic conjunctivitis
}

\author{
T Sumi, ${ }^{1}$ W Ishida, ${ }^{1}$ K Okumura, ${ }^{2}$ H Yagita, ${ }^{2}$ A Fukushima ${ }^{1}$
}

${ }^{1}$ Department of Ophthalmology and Visual Science, Kochi Medical School; ${ }^{2}$ Department of Immunology, Juntendo University School of Medicine

Correspondence to:

Dr A Fukushima, Department of Ophthalmology, Kochi Medical School, Kohasu, Oko-cho, Nankoku-city 783-8505, Japan; fukusima@kochi-u.ac.jp

Accepted 7 August 2008 Published Online First

9 September 2008

\section{ABSTRACT}

Background/aims: Tumour-necrosis-factor-related apoptosis-inducing ligand (TRAIL) plays a role in the development of allergic asthma. The study aimed to determine whether TRAIL also participates in the development of experimental allergic conjunctivitis (EC), another allergic disease model.

Methods: EC was induced in BALB/c mice by active immunisation with ragweed (RW) followed by RW challenge. To investigate whether TRAIL in the conjunctiva plays a role in the development of $\mathrm{EC}$, conjunctival TRAIL expression in EC-developing mice was evaluated by immunohistochemistry. Additionally, the effect of subconjunctival injection of recombinant TRAIL on conjunctival inflammation was examined. To investigate whether TRAIL expressed in systemic immunocompetent cells plays a role in the development of EC, anti-TRAIL blocking $A b$ or anti-TRAIL receptor agonistic Ab was intraperitoneally injected into EC-developing mice, and conjunctival eosinophil infiltration was evaluated.

Results: Conjunctival TRAIL expression was not increased by EC induction. Moreover, subconjunctival injection of TRAIL protein in naïve mice did not induce conjunctival inflammation. Thus, TRAIL in the conjunctiva is less likely to participate in the development of EC. Systemic treatment with anti-TRAIL blocking Ab in ECdeveloping mice did not affect the severity of $\mathrm{EC}$. However, systemic treatment during the induction phase of EC with an agonistic Ab for the TRAIL receptor significantly augmented the severity of EC and increased Ag-recall splenocyte IFN- $\gamma$ production in vitro.

Conclusions: These results indicate that TRAIL receptorexpressing cells in lymphoid organ participate in the development of EC.

Tumour necrosis factor (TNF)-related apoptosisinducing ligand (TRAIL) is a member of the TNF superfamily of cytokines. ${ }^{12}$ The physiological functions of TRAIL are not yet fully understood, but mouse gene knock-out studies indicate that this agent has an important role in anti-tumour surveillance by immune cells, mediates thymocyte apoptosis and is important in the induction of autoimmune diseases. ${ }^{3}$ In humans, TRAIL binds to two death-inducing receptors, DR4/TRAIL-R1 and DR5/TRAIL-R2/KILLER. TRAIL has also been shown to bind three decoy receptors, DcR1/ TRID/TRAIL-R3, DcR2/TRAIL-R4 and a soluble receptor osteoprotegerin (OPG) that was originally identified as a receptor for OPGL/RANKL. ${ }^{4}$ In the mouse, only one death-inducing receptor has been described that shares homology to human TRAILR2 (mouse DR5). ${ }^{4}$ TRAIL receptors are expressed in many different types of cells, including astrocytes and hepatocyotes in addition to immune cells. ${ }^{4}$

It has been reported that TRAIL participates in the regulation of different types of immune responses and inflammation. ${ }^{5}$ With regard to allergic diseases, patients with asthma show a significant increase in the levels of TRAIL in bronchoalveolar lavage fluid after allergen challenge. ${ }^{6}$ It was also demonstrated recently that TRAIL is abundantly expressed in the airway epithelium of mice developing experimental asthma. ${ }^{7}$ Furthermore, studies using TRAIL-deficient mice and short interfering RNA targeting TRAIL revealed that inhibition of TRAIL signalling impairs the production of CCL20 and the homing of myeloid dendritic cells and CCR6- and CD4expressing $\mathrm{T}$ cells to the airways. ${ }^{7}$ Thus, TRAIL appears to play a pivotal role in the development of asthma. However, to date, the roles TRAIL plays in other types of allergic diseases have not been investigated.

Allergic conjunctivitis (AC) is an allergic eye disease that ranges from mild forms such as seasonal AC to severe forms such as vernal keratoconjunctivitis (VKC). ${ }^{8}$ In VKC patients, eosinophils infiltrating into the conjunctiva affect the adjunct tissue cornea, sometimes leading to vision loss. ${ }^{9}$ Our group has investigated the mechanism that leads to VKC by inducing experimental allergic conjunctivitis (EC) in rats ${ }^{10-12}$ and mice. ${ }^{13-15}$ We found that Ag-specific $T$ cells, especially Th2 cells, play a crucial role in the infiltration of eosinophils into the conjunctiva during the development of EC.

Here, we sought to investigate whether TRAIL participates in the development of the allergic eye disease model EC.

\section{MATERIALS AND METHODS \\ Mice}

Inbred wild-type (WT) BALB/c mice were purchased from Japan SLC, Hamamatsu, Shizuoka, Japan. The mice were kept in specific pathogenfree conditions at the animal facility of Kochi Medical School, and age- and gender-matched mice were used when they were 6-12 weeks old. All research adhered to the Association for Research in Vision and Ophthalmology Statement for the Use of Animals in Ophthalmic and Vision Research.

\section{Reagents}

Short ragweed pollen (RW) was purchased from Polysciences (Warrington, PA). RW extract was obtained from LSL Co. Ltd (Tokyo). Aluminium 
hydroxide (alum) was purchased from Sigma (St Louis, MO). Generation and characterisation of the anti-mouse TRAIL blocking $\mathrm{Ab}$ (N2B2, rat $\operatorname{IgG})^{16}$ and the anti-mouse TRAIL receptor (DR5) agonistic Ab (MD5-1, hamuster IgG) ${ }^{17}$ have been described in our previous papers. These Abs for in vivo treatments were purified from ascites by using protein G columns. The Ab preparations contained less than $100 \mathrm{pg} / \mathrm{ml}$ endotoxin. Normal rat IgG (nrIgG) and normal hamster IgG (nhIgG) were obtained from MP Biomedicals, Aurora, $\mathrm{OH}$.

\section{EC induction by active immunisation and treatment with Abs}

RW adsorbed on alum was injected into the left hind-footpad and the tail base. Fifty microlitres of the emulsion (50 $\mu$ g of RW and $675 \mu \mathrm{g}$ of alum) was injected into each site. The mice were injected intraperitoneally with $200 \mu \mathrm{g}$ of purified anti-TRAIL, anti-DR5 $\mathrm{Ab}$, or control rat or hamster IgG ( $\mathrm{n}=10$ per group) on days $0,2,4,6$ and 8 after immunisation (induction phase). In a separate experiment, the actively immunised mice $(n=10$ per group) were injected intraperitoneally with $200 \mu \mathrm{g}$ of Ab only once $2 \mathrm{~h}$ before the RW challenge (effector phase). Ten days after active immunisation, the eyes of the immunised mice were challenged with RW in PBS ( $2 \mathrm{mg}$ in $10 \mu \mathrm{l}$ per eye). Twentyfour hours later, the eyes and spleens were harvested for histological analysis and cytokine assays or flow cytometric anlaysis, respectively.

\section{Induction of EC by adoptive transfer of in vitro-stimulated and Ab-treated RW-primed splenocytes}

Naive BALB/c mice were immunised with RW in their left hindfootpad and tail base, and were not treated with any Abs. Ten days later, their splenocytes were prepared and cultured with $5 \mu \mathrm{g} / \mathrm{ml} \mathrm{RW}$ extract as described previously ${ }^{13}{ }^{15}$ in the presence of $10 \mu \mathrm{g} / \mathrm{ml}$ anti-DR5 $\mathrm{Ab}$ or nhIgG ( $\mathrm{n}=5$ per group). After incubation for $72 \mathrm{~h}$ at $37^{\circ} \mathrm{C}$ in a humidified atmosphere with $5 \% \mathrm{CO}_{2}, 2 \times 10^{7}$ splenocytes were intraperitoneally injected into naïve mice. Four days after the splenocyte transfer, the eyes of the recipient mice were challenged with RW in PBS (2 mg in $10 \mu \mathrm{l}$ per eye). Twenty-four hours later, the eyes were harvested for histological analysis.

\section{Subconjunctival injection of recombinant mouse TRAIL}

Recombinant mouse (rm)TRAIL (Biomol, Plymouth Meeting, $\mathrm{PA} ; 1.56 \mu \mathrm{g}$ in $3 \mu \mathrm{l}$ of $0.1 \mathrm{mM}$ DTT/PBS) was injected into the subconjunctival space of naïve mice $(n=5)$. As a control, $3 \mu \mathrm{l}$ of $0.1 \mathrm{mM}$ DTT/PBS (vehicle) was injected $(n=5)$. Twenty-four hours after injection, the conjunctivas were harvested for histological analysis.

\section{Histological analysis}

The eyes including the conjunctivas were harvested and fixed in $10 \%$ buffered formalin. Vertical $2 \mu \mathrm{m}$ thick sections were cut and stained with Giemsa. Infiltrating eosinophils in the lamina propria mucosae of the tarsal and bulbar conjunctivas throughout each section were counted by two blinded observers. The sections counted were those of the central portion of the eye, which included the pupil and optic nerve head. The data are presented as averages (SEM) of all the mice examined.

\section{Immunohistochemistry}

Conjunctivas from actively immunised $\mathrm{BALB} / \mathrm{c}$ mice were harvested $24 \mathrm{~h}$ after RW challenge. In addition, conjunctivas from naive $\mathrm{BALB} / \mathrm{c}$ mice were collected. The conjunctivas were embedded in OCT compound (VWR, Suwanee, GA) and snap-frozen in liquid nitrogen. Vertical $4 \mu \mathrm{m}$ sections were prepared and fixed in methanol. Endogenous peroxidase activity was inhibited by incubation with $0.1 \% \mathrm{NaN}_{3}$ and $0.3 \% \mathrm{H}_{2} \mathrm{O}_{2}$ in distilled water for $10 \mathrm{~min}$ at room temperature. The samples were exposed to the anti-TRAIL Ab or anti-DR5 $\mathrm{Ab}$ for $30 \mathrm{~min}$ and then to appropriate biotinylated secondary $\mathrm{Ab}$ for another $30 \mathrm{~min}$. Ab binding was revealed by using an Avidin-Biotin-Complex kit (Vector Laboratories, Burlingame, CA) followed by development with 3,3'-diaminobenzidine tetrahydrochloride (Sigma).

\section{Measurement of cytokines in the culture supernatants}

$\mathrm{RBC}$-depleted splenocytes $\left(10^{7}\right.$ cells $\left./ \mathrm{ml}\right)$ were cultured for $48 \mathrm{~h}$ with RW extract $(25 \mu \mathrm{g} / \mathrm{ml})$ in 96 -well flat-bottom plates in a final volume of $0.2 \mathrm{ml}$ RPMI 1640 medium supplemented with $10 \%$ FCS and 2-ME. The levels of IL-4, IL-5, IL-12 and IFN- $\gamma$ that were produced were measured by using Luminex100 (Luminex, Austin, TX) and the Bio-Plex Cytokine Assay Panel (Bio-Rad Laboratories, Hercules, CA) according to the manufacturers' instructions.

\section{Flow-cytometric analysis}

RBC-depleted splenocytes from naïve mice or actively immunised and nrIgG-treated mice as described above were subjected to flow cytometric analysis to detect TRAIL- or DR5-expressing cells. To examine the effect of anti-DR5 Ab on splenocytes, RBC-depleted splenocytes from naïve mice were cultured as described above (following the method for splenocyte transfer) in the presence of either nhIgG or anti-DR5 Ab $(10 \mu \mathrm{g} / \mathrm{ml})$. Twenty-four hours later, the splenocytes were harvested and initially stained with biotin-labelled anti-CD4 Ab or anti-CD8 $\mathrm{Ab}$ followed by streptavidin-PE staining. Then, these cells were stained with annexin V-fluorescein isothiocyanate according to the manufacturer's recommendations (BioVision, Mountain View, CA). Dead cells were excluded by 7 -amino-actinomycin $\mathrm{D}$ (7-AAD). The cells were then analysed on a FACScan (Becton Dickinson, Mountain View, CA), and the data were acquired and analysed by using CellQuest software.

\section{Statistical analysis}

Differences between the Ab-treated and control IgG-treated mice in terms of their cytokine production and infiltrating eosinophil numbers were tested for significance using the Student $t$ test. $p$ Values less than 0.05 were considered significant.

\section{RESULTS}

TRAIL is not induced in the conjunctiva by the induction of EC A previous report showed that TRAIL was induced in the airway epithelium by the induction of experimental asthma. To examine whether this is also the case in EC, an immunohistochemical analysis was performed to detect TRAIL expression in the conjunctiva. TRAIL expression was barely detected in either naïve or EC-developing conjunctivas (fig 1A). Similarly, expression of DR5 was hardly detectable in the conjunctiva from either naïve or EC-developing mice (data not shown).

\section{Subconjunctival injection of rmTRAIL does not induce conjunctival inflammation}

A previous report demonstrated that intratracheal administration of rmTRAIL in naïve animals induced airway hyperresponsiveness and inflammation. ${ }^{7}$ We thus asked whether injecting rmTRAIL into the subconjunctival space of naïve mice would 
induce conjunctival inflammation. However, there was no difference between the rmTRAIL-injected and vehicle-injected groups in terms of conjunctival eosinophil infiltration when it was evaluated $24 \mathrm{~h}$ later (fig 1B). T cells, but not B cells and neutrophils, also infiltrated into the conjunctiva (data not shown).

\section{Systemic blockade of TRAIL does not affect EC or RW-recall immune responses}

The above findings suggest that TRAIL in the conjunctiva is less likely to be involved in the development of EC. Therefore, we next investigated whether systemic TRAIL participates in the development of EC. For this, anti-TRAIL blocking Ab was injected intraperitoneally into mice that had been immunised with RW and were then challenged with RW in eye-drops. AntiTRAIL Ab was either injected during the induction phase (five times every other day from the day of immunisation) or during the effector phase ( $2 \mathrm{~h}$ prior to RW challenge). Anti-TRAIL Ab treatment during the induction and effector phases slightly suppressed (fig 2A) and elevated (fig 2C) the severity of EC, respectively, but these changes did not achieve statistical significance. The RW-recall cytokine production of splenocytes from these mice did not vary, regardless of when, during the disease process, the mice had been treated with anti-TRAIL Ab (fig 2B,D). Importantly, TRAIL-expressing cells constituted less than $1 \%$ of the whole splenocyte population and their TRAIL expression levels were minute (percentage positive: $0.14(0.00)$ in naïve mice and $0.60(0.04)$ in nrIgG-treated actively immunised group).

\section{Agonistic stimulation of TRAIL receptor affects EC and immune responses}

To investigate whether forced stimulation of systemic TRAIL receptor affects the severity of $\mathrm{EC}, \mathrm{RW}$-immunised mice were injected intraperitoneally with anti-DR5 $\mathrm{Ab}$, an agonistic $\mathrm{Ab}$ for mouse TRAIL receptor, during the induction phase (5 times every other day from the day of immunisation) or the effector phase ( $2 \mathrm{~h}$ prior to RW challenge). Anti-DR5 Ab treatment during the induction phase significantly elevated the severity of EC, as indicated by conjunctival eosinophil numbers (fig 3A). Anti-DR5 Ab treatment also increased conjunctival eosinophil infiltration when delivered during the effector phase (fig 3C), although this difference did not reach statistical significance. Anti-DR5 Ab treatment during the induction phase also significantly upregulated RW-recall IFN- $\gamma$ production, while the other cytokines tested were not affected (fig 3B). In contrast, effector phase treatment with anti-DR5 Ab did not affect the cytokine production (fig 3D). Similar to the data of TRAIL, TRAIL receptor expression levels in splenocytes were minute (percentage positive: $0.21(0.02)$ in naïve mice 0.67 (0.09) in nhIgG-treated actively immunised group).

\section{Stimulation of RW-primed splenocytes with anti-DR5 Ab increased IL-5 production and EC-inducing capability but suppressed IFN- $\gamma$ production}

Next, we investigated how in vitro treatment with anti-DR5 Ab affects RW-primed splenocyte responses. RW-primed splenocytes that were stimulated with RW extract together with antiDR5 Ab produced significantly higher amounts of IL-5 and significantly lower amounts of IFN- $\gamma$ than those that were cultured with the control nhIgG Ab (fig 4A). Furthermore, the adoptive transfer into naïve mice of RW-primed splenoctyes stimulated in vitro with RW-extract and anti-DR5 Ab, followed by RW challenge 4 days later, induced significantly more severe EC than adoptive transfer of the nhIgG-treated splenocytes (fig 4B).

\section{Stimulation of naïve splenocytes with anti-DR5 Ab increased apoptotic cells}

Finally, we investigated whether anti-DR5 Ab induces apoptosis of splenocytes. Naïve splenocytes were cultured for $24 \mathrm{~h}$ in the presence of either anti-DR5 Ab or nhIgG. These splenocytes were subjected to flow-cytometric analysis to determine annexin $\mathrm{V}$-positive cells. Most of splenocytes were 7-AADnegative. In both $\mathrm{CD}^{+}$and $\mathrm{CD}^{+} \mathrm{T}$ cell compartments, annexin $\mathrm{V}$-positive cells apparently increased in the presence of anti-DR5 Ab compared with nhIgG (fig 5).

\section{DISCUSSION}

While a previous report has shown that TRAIL is induced in airway epithelium by the induction of experimental asthma, ${ }^{7}$ the induction of EC did not induce TRAIL in the conjunctiva. It
A

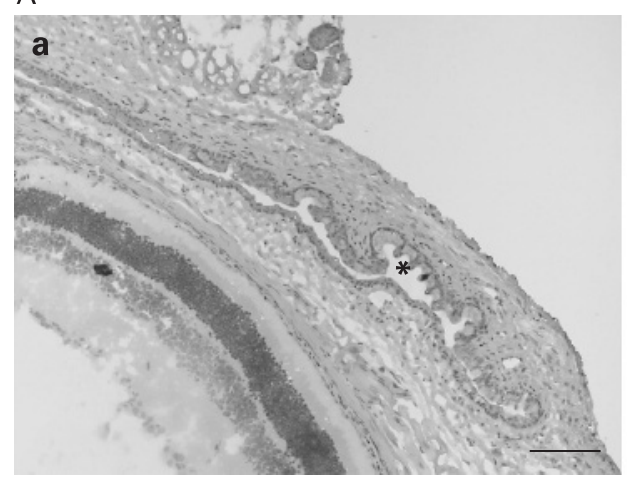

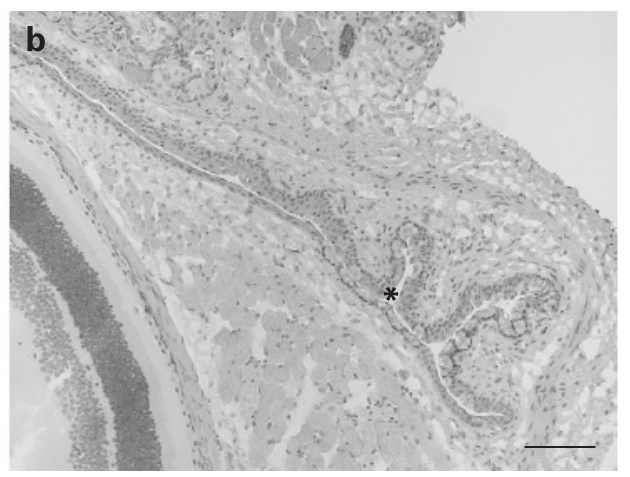

B

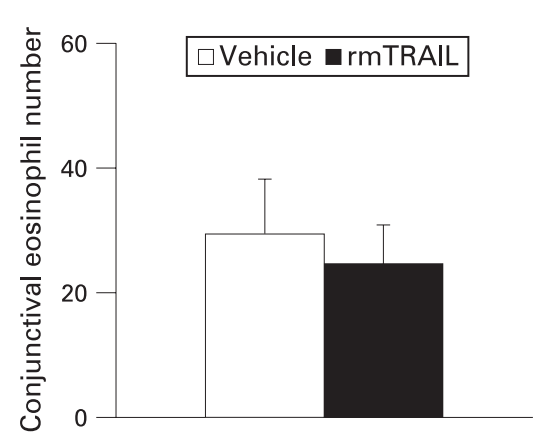

Figure 1 Roles of tumour necrosis factor-related apoptosis-inducing ligand (TRAIL) in the conjunctiva. (A) Expression of TRAIL in the conjunctiva. Conjunctivas from naïve (a) and EC-developing (b) mice were harvested to evaluate TRAIL expression by immunohistochemical analysis. Minute amounts of TRAIL protein were detected in the conjunctival epithelial layers, but there was no obvious difference between naïve and EC-developing mice. ${ }^{*}$ Conjunctival epithelium. Bar $=100 \mu \mathrm{m}$. One representative of three samples is shown. (B) Effects of subconjunctival injection of recombinant mouse TRAIL (rmTRAIL). rmTRAIL or vehicle was injected into the subconjunctival space of naïve BALB/c mice. Twenty-four hours later, the conjunctivas were harvested for histological analysis to determine conjunctival eosinophil numbers. A significant difference between the two groups was not observed. 
Figure 2 Effects of treatment with a blocking anti-TRAIL blocking $A b$ on the severity of experimental allergic conjunctivitis (EC) and splenocyte cytokine production. EC was induced in $\mathrm{BALB} / \mathrm{c}$ mice by active immunisation with ragweed (RW) followed by RW challenge. Twenty-four hours after RW challenge, the conjunctivas and spleens were harvested for histological analysis to determine conjunctival eosinophil numbers $(A, C)$ and $A g$-recall splenocyte cytokine production $(B, D)$, respectively. The blocking anti-TRAIL Ab or control nrlgG was injected intraperitoneally during the induction phase $(A, B)$ or the effector phase $(C, D)$. The anti-TRAIL Aband nrlgG-treated groups did not differ significantly in conjunctival eosinophil numbers $(A, C)$ and splenocyte cytokine production $(B, D)$, regardless of when in the disease process they were treated. In (A), conjunctival eosinophil numbers in naïve and RW-challenged BALB/c mice were presented as a control.
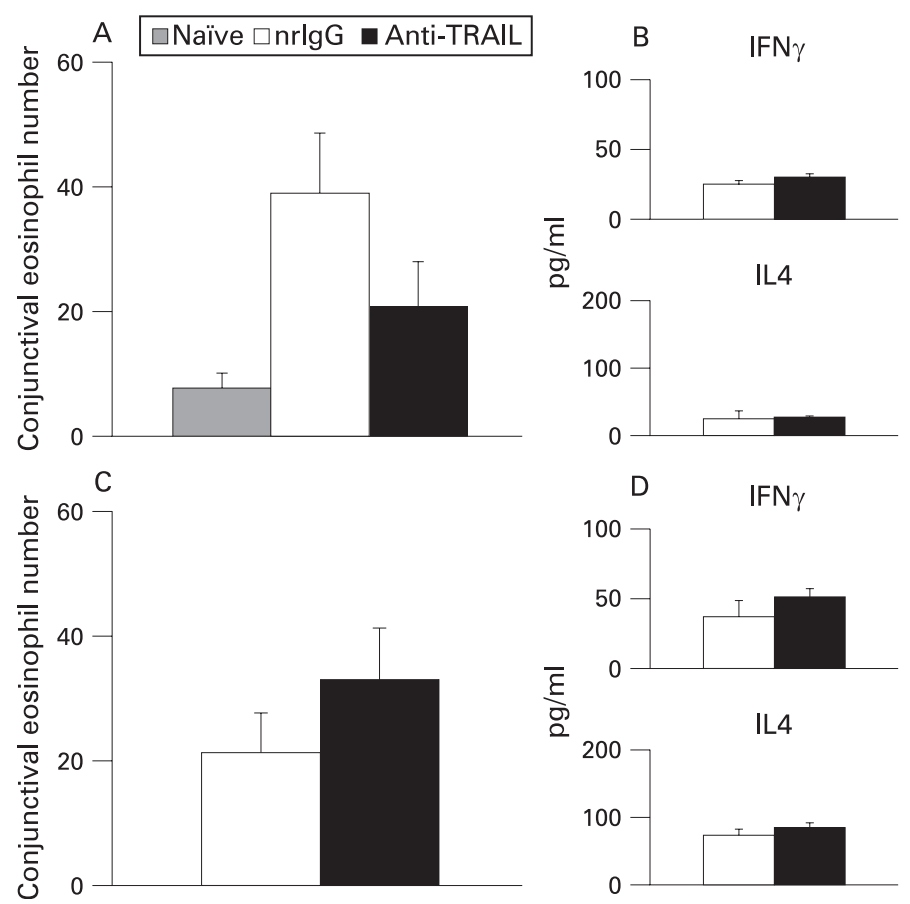

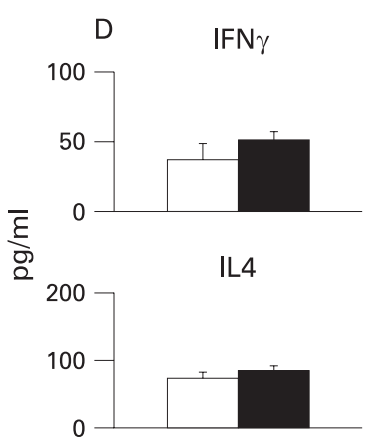

IL5

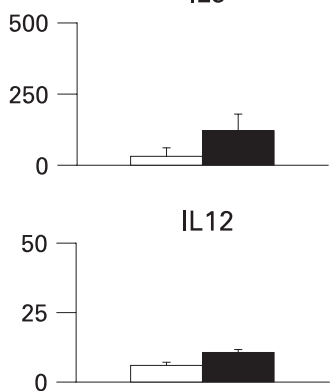

IL5

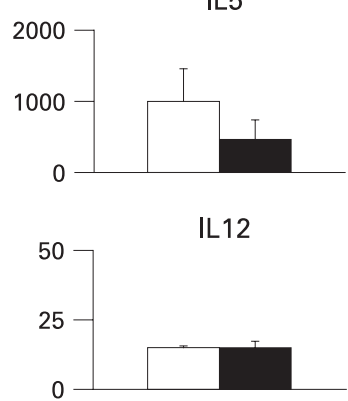

should be noted that the Ab we used for immunohistochemical analysis was the same as that used in the previous report. ${ }^{7}$ Moreover, while it was reported that the intratracheal administration of rmTRAIL in naive animals induces pathognomic features of asthma, ${ }^{7}$ significant conjunctival inflammation was not observed after subconjunctival injection of rmTRAIL. These observations together suggest that it is less likely that TRAIL in the conjunctiva plays a critical role in the development of EC.

We next investigated whether systemic TRAIL participates in the development of EC. Blockade of TRAIL during the induction or effector phase did not affect the severity of EC. We also found by flow-cytometric analysis that TRAIL expression levels were minute. This may be why treatment with the blocking antiTRAIL Ab did not significantly affect the severity of EC and splenocyte cytokine production. However, agonistic stimulation of TRAIL receptor by anti-DR5 Ab during the induction phase significantly increased the severity of EC and splenocyte IFN- $\gamma$ production, although TRAIL receptor expression in splenocytes was at a similar level to that of TRAIL. Thus, it appears that TRAIL receptor-expressing cells affect the severity of EC. Indeed, treatment of splenocytes with anti-DR5 Ab in vitro apparently increased apoptotic cells.

A notable finding was that in vitro Ag-recall splenocyte IFN- $\gamma$ production was upregulated by in vivo treatment with
Figure 3 Effects of agonistic anti-DR5 Ab treatment on the severity of experimental allergic conjunctivitis (EC) and splenocyte cytokine production. EC was induced in BALB/c mice by active immunisation with ragweed (RW) followed by RW challenge. Twenty-four hours after RW challenge, the conjunctivas and spleens were harvested for histological analysis to determine conjunctival eosinophil numbers $(A, C)$ and Ag-recall splenocyte cytokine production $(B, D)$, respectively. Anti-DR5 agonistic $A b$ or control nhlgG was injected intraperitoneally during the induction phase $(A, B)$ or the effector phase $(C, D)$. When the treatment was conducted during the induction phase, conjunctival eosinophil numbers $(A)$ and splenocyte IFN- $\gamma$ production (B) were significantly higher in the anti-DR5 Abtreated group than the nhlgG-treated group. ${ }^{*} p<0.05$. No significant difference between the two groups was noted when the treatment was conducted during the effector phase $(C, D)$.
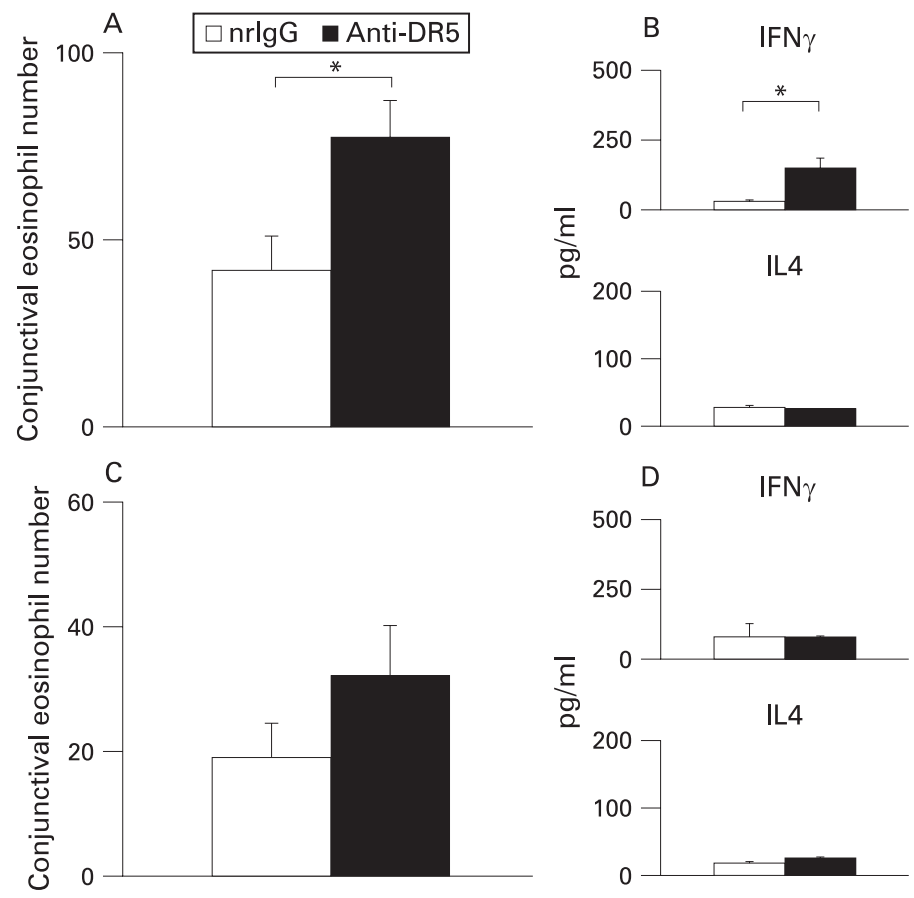

IL5

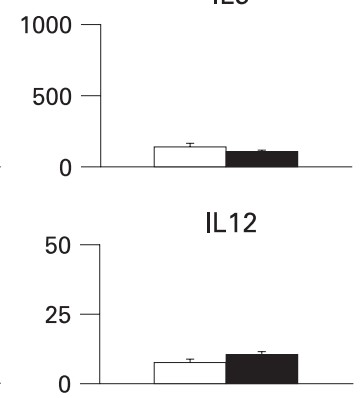

IL5

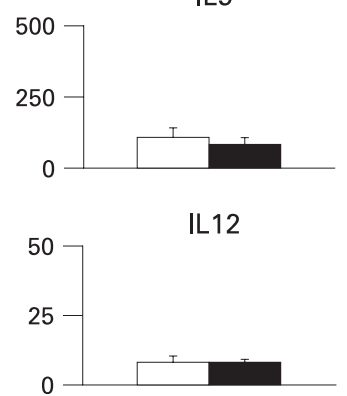



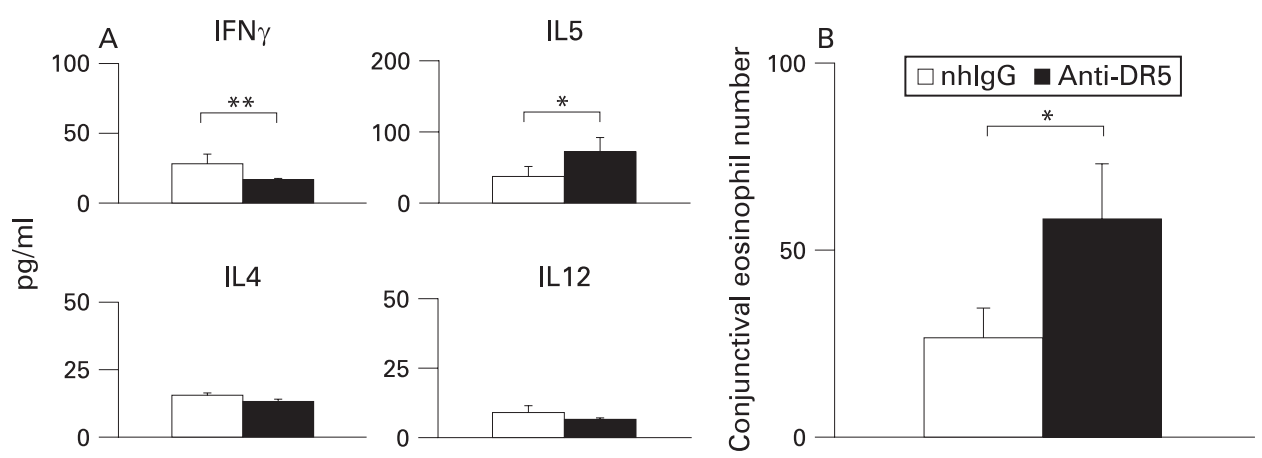

Figure 4 Effects of in vitro treatment with agonistic anti-DR5 Ab on Ag-recall splenocyte responses. BALB/c mice were immunised with ragweed (RW) in alum. Ten days later, their spleens were harvested, and the splenocytes were stimulated with RW extract in vitro together with anti-DR5 Ab or nhlgG. Forty-eight hours later, culture supernatants were collected for cytokine assays (A). After 3 days of culture, the stimulated splenocytes were collected and transferred into naïve BALB/c mice by intraperitoneal injection. Four days later, the recipient mice were challenged with RW in eye-drops, and $24 \mathrm{~h}$ later, the conjunctivas were harvested for histological analysis to determine conjunctival eosinophil numbers (B). Significantly higher amounts of IL-5 and significantly lower amounts of IFN- $\gamma$ were produced by splenocytes treated in vitro with anti-DR5 Ab compared with the control nhlgG Ab (A). Adoptive transfer of splenoctyes treated in vitro with anti-DR5 Ab induced significantly more severe experimental allergic conjunctivitis than those with nhlgG (B). ${ }^{* *} p<0.01,{ }^{*} p<0.05$.

anti-DR5 Ab. With regard to TRAIL expression in T cells, it has been reported that the activation-induced expression of TRAIL was exclusively observed in Th2 clones and primary helper T cells that have differentiated under Th2 conditions. ${ }^{18}$ It has also been reported that Th2 cells were more resistant to TRAILinduced apoptosis than Th1 cells. ${ }^{18}$ However, roles TRAIL receptor plays in Th1/Th2 balance have not been demonstrated. Stimulation of TRAIL receptor of splenocytes in vitro with antiDR5 Ab upregulated IL-5 production but suppressed IFN- $\gamma$ production. In contrast, the stimulation of TRAIL receptor by systemic in vivo treatment with anti-DR5 Ab increased splenocyte IFN- $\gamma$ production. The reason for this discrepancy is unclear at this point. TRAIL expression is not only restricted to $T$ cells, as it is also observed in eosinophils ${ }^{19}$ and some mouse liver natural killer cells. ${ }^{20}$ Additionally, it was reported that treatment with anti-DR5 $\mathrm{Ab}$ recruits $\mathrm{Fc}$ receptor-expressing innate immune cells and exhibits anti-tumour effects against TRAIL-sensitive tumour cells in vivo. ${ }^{17}$ It is possible that the effects of agonistic stimulation of TRAIL receptor by intraperitoneal injection of anti-DR5 Ab affected innate immune cells as well as $\mathrm{T}$ cells, thereby elevating splenocyte IFN- $\gamma$ production.

How did the upregulated IFN- $\gamma$ production induced by antiDR5 Ab treatment in vivo increase the severity of EC? We have previously shown that endogenous IFN- $\gamma$ promotes the development of $\mathrm{EC}$ in the BALB/c background but not in the C57BL/ 6 background. ${ }^{15}$ Furthermore, it was also demonstrated that IFN- $\gamma$ participated in the development of allergic conjunctivitis during the effector phase in $\mathrm{BALB} / \mathrm{c}$ mice. ${ }^{21}$ The latter report also indicated that IFN- $\gamma$ induced vascular endothelial adhesion molecule (VCAM)-1 expression in the conjunctival vasculature, thus inducing infiltration of inflammatory cells into the conjunctiva. ${ }^{21}$ These facts are in good agreement with our data showing that anti-DR5 Ab treatment in vivo increased IFN- $\gamma$ production and augmented the severity of $\mathrm{EC}$ in $\mathrm{BALB} / \mathrm{c}$ mice. In contrast, we have also previously demonstrated that stimulation of Ag-specific $\mathrm{T}$ cells in the presence of excessive IL- 4 and IFN- $\gamma$ significantly upregulated and attenuated the ECinducing capability (conjunctival eosinophil infiltration) of these $\mathrm{T}$ cells, respectively. ${ }^{22}$ This is also in good agreement with the data that anti-DR5 Ab treatment in vitro significantly attenuated IFN- $\gamma$ production but significantly upregulated EC-inducing capability.
Endogenous TRAIL is less likely to participate in the development of EC but forced stimulation of TRAIL receptor by anti-DR $5 \mathrm{Ab}$ significantly augmented the severity of EC. As mentioned in the Introduction, the ligand of DR5 is TRAIL alone; however, the ligand of TRAIL is not restricted to DR5 but also includes decoy receptors. Therefore, the effect of treatment with anti-DR5 Ab is different from that of endogenous TRAIL which also affects decoy receptors. It may be possible that the distribution and abundance of each TRAIL receptor are different
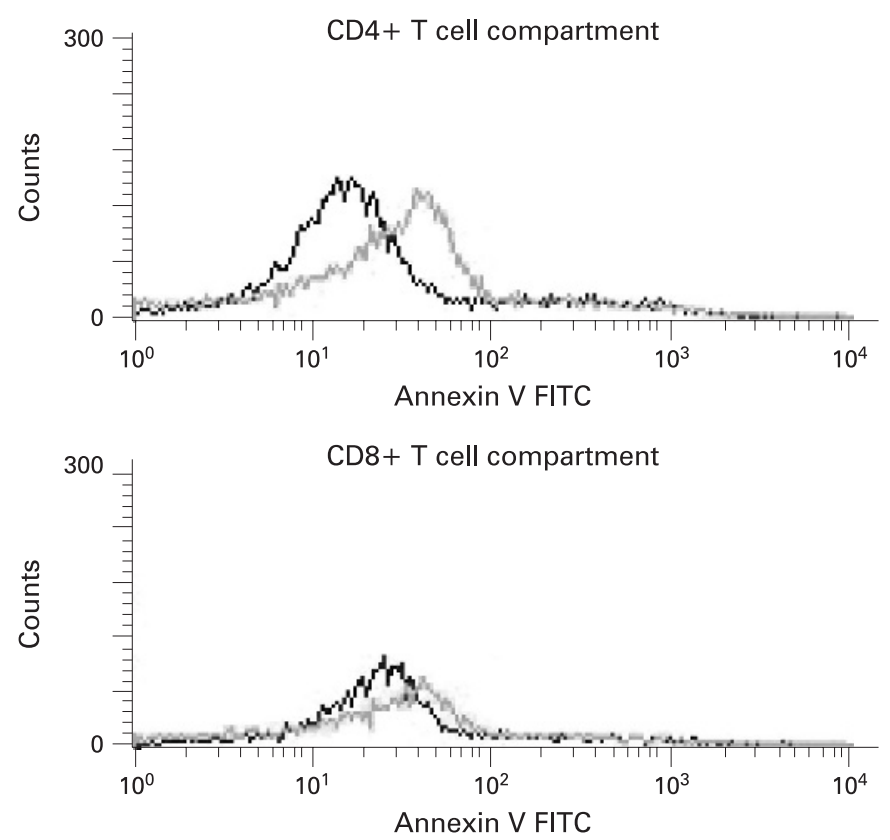

Figure 5 Effects of in vitro treatment with agonistic anti-DR5 Ab on apoptosis of splenocytes. Splenocytes were harvested from naïve BALB/ $c$ mice were stimulated in vitro with anti-DR5 Ab or nhlgG. Twenty-four hours later, these splenocytes were subjected to flow-cytometric analysis to determine the frequencies of annexin $\mathrm{V}$-positive and 7-aminoactinomycin $\mathrm{D}$ (7-AAD)-negative cells in $\mathrm{CD}^{+}$and $\mathrm{CD} 8^{+} \mathrm{T}$ cell compartments. Most of the splenocytes were negative for 7-AAD. One representative histogram, based on the data, is shown. Grey line, antiDR5 Ab treated splenocytes; black line, nhlgG treated splenocytes; FITC, fluorescein isothiocyanate. 
between the airways and conjunctiva. This may be one reason why TRAIL plays a role in the development of experimental airway inflammation, but not in EC.

In conclusion, TRAIL is less likely to play a significant role in the development of EC, whereas TRAIL receptor expressing cells in lymphoid organ participate in the development of EC. Future studies on TRAIL receptor expression and stimulation in draining lymph nodes may shed more light on the role of these receptors in EC.

Acknowledgements: We thank A Ojima and M Kajisako for their excellent technical help.

Funding: This work was supported in part by Grant-in-Aid for Scientific Research from the Ministry of Education, Culture, Sports, Science, and Technology, Japan (AF).

Competing interests: None.

\section{REFERENCES}

1. Wiley SR, Schooley K, Smolak PJ, et al. Identification and characterization of a new member of the TNF family that induces apoptosis. Immunity 1995;3:673-82.

2. Locksley RM, Killeen N, Lenardo MJ. The TNF and TNF receptor superfamilies: integrating mammalian biology. Cell 2001;104:487-501.

3. Carlo-Stella C, Lavazza C, Locatelli A, et al. Targeting TRAIL agonistic receptors for cancer therapy. Clin Cancer Res 2007;13:2313-17.

4. Cretney E, Takeda K, Smyth MJ. Cancer: novel therapeutic strategies that exploit the TNF-related apoptosis-inducing ligand (TRAIL)/TRAIL receptor pathway. Int J Biochem Cell Biol 2007;39:280-6.

5. Aggarwal BB, Shishodia S, Ashikawa K, et al. The role of TNF and its family members in inflammation and cancer: lessons from gene deletion. Curr Drug Targets Inflamm Allergy 2002;1:327-41.

6. Robertson NM, Zangrilli JG, Steplewski A, et al. Differential expression of TRAIL and TRAIL receptors in allergic asthmatics following segmental antigen challenge: evidence for a role of TRAIL in eosinophil survival. J Immunol 2002;169:5986-96.

7. Weckmann M, Collison A, Simpson JL, et al. Critical link between TRAIL and CCL20 for the activation of $\mathrm{T}_{\mathrm{H}} 2$ cells and the expression of allergic airway disease. Nat Med 2007:13:1308-15.

8. Trocme SD, Sra KK. Spectrum of ocular allergy. Curr Opin Allergy Clin Immunol 2002;2:423-7.
9. Hingorani M, Calder V, Jolly G, et al. Eosinophil surface antigen expression and cytokine production vary in different ocular allergic diseases. J Allergy Clin Immunol 1998;102:821-30.

10. Fukushima A, Yoshida $\mathrm{H}$, Iwamoto $\mathrm{H}$, et al. The role of cellular immunity both in the induction and effector phases of experimental allergic blepharoconjunctivitis (EAC) in rats. Exp Eye Res 1997;65:631-7.

11. Yoshida 0, Yoshida $\mathrm{H}$, Iwamoto $\mathrm{H}$, et al. Genetic background determines the nature of immune responses and experimental immune-mediated blepharoconjunctivitis (EC). Curr Eye Res 1999;18:117-24.

12. Fukushima A, Ozaki A, Fukata K, et al. Ag-specific recognition, activation, and effector function of $T$ cells in the conjunctiva with experimental immune-mediated blepharoconjunctivitis. Invest Ophthalmol Vis Sci 2003;44:4366-74.

13. Fukushima A, Ozaki A, Jian Z, et al. Dissection of antigen-specific humoral and cellular immune responses for the development of experimental immune-mediated blepharoconjunctivitis in C57BL/6 mice. Curr Eye Res 2005;30:241-8.

14. Ozaki A, Seki Y, Fukushima A, et al. The control of allergic conjunctivitis by suppressor of cytokine signaling (SOCS)3 and SOCS5 in a murine model. J Immunol 2005;175:5489-97.

15. Fukushima A, Yamaguchi T, Ishida W, et al. Genetic background determines susceptibility to experimental immune-mediated blepharoconjunctivitis: comparison of Balb/c and C57BL/6 mice. Exp Eye Res 2006;82:210-18.

16. Kayagaki N, Yamaguchi N, Nakayama M, et al. Expression and function of TNFrelated apoptosis-inducing ligand on murine activated NK cells. J Immunol 1999;163:1906-13.

17. Takeda K, Yamaguchi N, Akiba H, et al. Induction of tumor-specific T cell immunity by anti-DR5 antibody therapy. J Exp Med 2004;199:437-48.

18. Greeneltch KM, Kelly-Welch AE, Shi Y, et al. Chronic morphine treatment promotes specific Th2 cytokine production by murine T cells in vitro via a Fas/Fas liganddependent mechanism. J Immunol 2005;175:4999-5005.

19. Robertson NM, Zangrilli JG, Steplewski A, et al. Differential expression of TRAIL and TRAIL receptors in allergic asthmatics following segmental antigen challenge: evidence for a role of TRAIL in eosinophil survival. J Immunol 2002;169:5986-96.

20. Smyth MJ, Cretney E, Takeda K, et al. Tumor necrosis factor-related apoptosisinducing ligand (TRAIL) contributes to interferon $\gamma$-dependent natural killer cell protection from tumor metastasis. J Exp Med 2001;193:661-70.

21. Stern ME, Siemasko K, Gao J, et al. Role of interferon-gamma in a mouse model of allergic conjunctivitis. Invest Ophthalmol Vis Sci 2005;46:3239-46.

22. Ozaki A, Fukushima A, Fukata K, et al. Effects of IL-4 and IL-12 on experimental immune-mediated blepharoconjunctivitis in Brown Norway rats. Clin Exp Immunol 2000;122:28-34. 